ESAIM: PROCEEDINGS, December 2012, Vol. 38, p. 376-386

F. Coquel, M. Gutnic, P. Helluy, F. Lagoutière, C. Rohde, N. Seguin, Editors

\title{
A FINITE VOLUME METHOD FOR DENSITY DRIVEN FLOWS IN POROUS MEDIA
}

\author{
Danielle Hilhorst ${ }^{1}$, Huy Cuong Vu Do ${ }^{2}$ and Yushan Wang ${ }^{3}$
}

\begin{abstract}
In this paper, we apply a semi-implicit finite volume method for the numerical simulation of density driven flows in porous media; this amounts to solving a nonlinear convection-diffusion parabolic equation for the concentration coupled with an elliptic equation for the pressure. We compute the solutions for two specific problems: a problem involving a rotating interface between salt and fresh water and the classical but difficult Henry's problem. All solutions are compared to results obtained by running FEflow, a commercial software package for the simulation of groundwater flow, mass and heat transfer in porous media.
\end{abstract}

\section{INTRODUCTION}

We describe here results which have been obtained in the context of an exploratory project of CNRS (PEPS ECODEVA) on the numerical simulation of flows with variable density for the production of lithium batteries. More precisely, the purpose of this project is related to the exploitation of lithium deposits in salt lakes, also known as "Salars". In recent years, lithium has become a strategic element for industrial countries because it is the basic element of lithium-ion batteries used for hybrid and electric vehicles. Therefore its production has become of high interest for all major groups involved in the car industry as well as suppliers of these groups. Currently the largest deposit in the world is the Salar Uyuni, in the department of Potosí, in South-West Bolivia. This deposit represents a third of the world resources. In March 2008, Bolivia has authorized the exploitation, however reserving this right to its nationals. Chile has the second largest deposit with the Salar Atacama and it has become the world's largest exporter since 1997, with the German company Chemettal as main operator. Argentina also has a lithium deposit, the Salar Hombre Muerto, which is located in the North-West of the country. Other salar areas of the Altiplano of Argentina provide mining exploitation concessions to foreign companies, among whom European groups.

Other deposits are exploited, including salt lakes in Tibet as well as mines in Australia, Russia and the United States. They are not accessible to European operators. The largest deposits are either clusters of crystallized salt (solid) or lenses of supersaturated salt water created by evaporation under endorheic conditions (which are not led to a superficial network reaching the sea). The latter type of deposit is that of salars of the Andean altiplano. Rational exploitation implies mastering these special aqueous flows whose density depends on the concentration of salts (lithium included). An operating technique consists in sweeping the reservoir with fresh

${ }^{1}$ Laboratoire de Mathématiques, CNRS et Université de Paris-Sud (Bâtiment 425)

2 Early Stage Researcher of the European Marie Curie ITN Project FIRST, Laboratoire de Mathématiques, Université de Paris-Sud (Bâtiment 425)

${ }^{3}$ Laboratoire de Recherche en Informatique, Université de Paris-Sud (Bâtiment 650)

(C) EDP Sciences, SMAI 2012 
water in order to obtain a maximal recovery without earthworks and with a minimal impact on fluid levels, and thus a minimal impact on the environment. This explains the need of implementing research methodologies and techniques from hydrogeology. The purpose here is to extract salt water which contains lithium. In a later stage, the lithium will be separated from the salt water.

From a mathematical viewpoint, it amounts to study a coupled system describing the interaction between flow and transport in a porous medium, whose density $\rho$ is a strictly increasing function of the concentration $c$ of the salt water; typically we have that $\rho(c)=\rho_{0}(1+\alpha c)$. More specifically, we solve a nonlinear parabolic convection-diffusion equation for the concentration coupled with an elliptic equation for the pressure, which is derived from Darcy's law

$$
\begin{cases}\Phi \frac{\partial(\rho(c) c)}{\partial t}+\nabla \cdot(\mathbf{q} \rho(c) c-\rho(c) D \nabla c)=0, & \text { in } \Omega \times(0, T] \\ \Phi \frac{\partial \rho(c)}{\partial t}+\nabla \cdot(\mathbf{q} \rho(c))=0, & \text { in } \Omega \times(0, T] \\ \mathbf{q}=-\frac{k}{\mu}(\nabla p-\rho(c) \mathbf{g}), & \text { in } \Omega \times(0, T]\end{cases}
$$

together with suitable boundary conditions. The porosity $\Phi$ is the fraction of the voids (empty spaces) over the total volume. In the third equation of (1), namely, Darcy's law, q is the flux (discharge per unit area), $p$ is the pressure, $k$ is the permeability, $\mu$ is the dynamic viscosity, and $\mathbf{g}$ is the gravity.

The organization of this paper is as follows. In Section 1, we present the numerical algorithm which is applied to solve System (1). It is based upon the standard finite volume method for the spatial discretization and a semi-implicit scheme for the time discretization [9], [6], [7], [8]. Because of the form of the velocity vector q, we simultaneously use two upwind directions for the discretization of the convection terms. In Section 2 , we describe Henry's problem, together with suitable boundary conditions. The main difficulty is that we have to compute pressure and velocity, in a case that the velocity changes direction through one of the boundaries. In Section 3, we study the time evolution of the interface between fresh and salt water in an aquifer in a case that the interface slowly rotates before reaching a stable equilibrium where the fresh water lies above the salt water. A technical difficulty is that the pressure is not uniquely defined since Neumann boundary conditions are prescribed on the boundaries. This leads us to slightly modify System (1) by adding a small parabolic term to the pressure equation. All the solutions which we compute throughout this paper are compared to results obtained by means of FEflow, a commercial software for the simulation of groundwater flow and mass and heat transfer in porous media, which is based upon the finite element method. Finally, we present some conclusions in Section 4.

We refer to [5], [11], [2], [4] for modelling aspects. System (1) has already been solved in [10] by means of a vertex centered finite volume method as well as by the mixed finite element method. They directly applied their numerical method to a real case, while we perform a rather detailed study of the application of our numerical scheme to two rather intricate test cases.

\section{The Finite Volume DiscRetization}

In this section, we discretize System (1)

$$
\begin{cases}\Phi \frac{\partial(\rho(c) c)}{\partial t}+\nabla \cdot(\mathbf{q} \rho(c) c-\rho(c) D \nabla c)=0, & \text { in } \Omega \times(0, T] \\ \Phi \frac{\partial \rho(c)}{\partial t}+\nabla \cdot(\mathbf{q} \rho(c))=0, & \text { in } \Omega \times(0, T] \\ \mathbf{q}=-\frac{k}{\mu}(\nabla p-\rho(c) \mathbf{g}), & \text { in } \Omega \times(0, T]\end{cases}
$$


together with the boundary conditions

$$
\begin{cases}c=c_{D}(\mathbf{x}, t), & (x, t) \in \partial \Omega_{D}^{c} \times(0, T], \\ p=p_{D}(\mathbf{x}, t), & (x, t) \in \partial \Omega_{D}^{p} \times(0, T], \\ \frac{\partial c}{\partial n}=\bar{c}_{N}(\mathbf{x}, t), & (x, t) \in \partial \Omega_{N}^{c} \times(0, T], \\ \mathbf{q} \cdot \mathbf{n}=\bar{q}_{N}(\mathbf{x}, t), & (x, t) \in \partial \Omega_{N}^{p} \times(0, T],\end{cases}
$$

where $\partial \Omega=\partial \Omega_{D}^{c} \bigcup \partial \Omega_{N}^{c}=\partial \Omega_{D}^{p} \bigcup \partial \Omega_{N}^{p}$ with $\partial \Omega_{D}^{c}, \partial \Omega_{D}^{p}$ the Dirichlet boundaries and $\partial \Omega_{N}^{c}, \partial \Omega_{N}^{p}$ the Neumann boundaries for concentration and pressure respectively. The initial conditions are given by

$$
\begin{cases}c(\mathbf{x}, 0)=c_{0}(\mathbf{x}), & \text { for all } \mathbf{x} \in \Omega, \\ p(\mathbf{x}, 0)=p_{0}(\mathbf{x}), & \text { for all } \mathbf{x} \in \Omega .\end{cases}
$$

Next we introduce some notations related to the space and time discretizations.

Space discretization: Let $\Omega$ be an open bounded polygonal subset of $\mathbb{R}^{N}$ and $\mathcal{T}$ be a mesh of $\Omega$; for all $K \overline{\in \mathcal{T} \text {, we denote by }|K|}$ the measure of $K$ in $\mathbb{R}^{N}$. The elements of $\mathcal{T}$ will be called control volumes in what follows. For any $(K, L) \in \mathcal{T}^{2}$ with $K \neq L$, we denote by $\sigma_{K L}=\bar{K} \cap \bar{L}$ their common interface; it is included in a hyperplane of $\mathbb{R}^{N}$, which does not intersect $K$ nor $L$. Then $\left|\sigma_{K L}\right|$ denotes the measure of $\sigma_{K L}$ for the Lebesgue measure of the hyperplane, and $\mathbf{n}_{K L}$ denotes the unit vector normal to $\sigma_{K L}$, oriented from $K$ to $L$. The set of boundary control volumes is denoted by $\partial \mathcal{T}=\{\partial K \cap \partial \Omega, K \in \mathcal{T}\}$ and for all $K \in \partial \mathcal{T}$, we denote $\sigma_{K}$ the intersection of the boundary of $K$ and the boundary of $\Omega$, i.e. $\sigma_{K}=\partial K \cap \partial \Omega$.

We denote by $\mathcal{E}$ the set of edges $\sigma$ of the control volumes. For any $K \in \mathcal{T}$, we define the subset $\mathcal{E}_{K}$ of $\mathcal{E}$ as the set of edges of the volume element $K$. Then $\mathcal{E}=\bigcup_{K \in \mathcal{T}} \mathcal{E}_{K}$. For all $K \in \mathcal{T}$, we define the set of neighbors of $K$ as $\mathcal{N}_{K}=\left\{L \in \mathcal{T},(K, L) \in \mathcal{T}^{2}, L \neq K,\left|\sigma_{K L}\right| \neq 0\right\}$.

We suppose that there exists a family of points $\mathbf{x}_{K} \in \Omega$ such that

$$
\begin{cases}\mathbf{x}_{K} \in K, & \text { for all } K \in \mathcal{T}, \\ \frac{\mathbf{x}_{L}-\mathbf{x}_{K}}{d_{K L}}=\mathbf{n}_{K L}, & \text { for all }(K, L) \in \mathcal{T}^{2},\end{cases}
$$

where $d_{K L}=\left|\mathbf{x}_{L}-\mathbf{x}_{K}\right|$. For all $K \in \mathcal{T}$ and $\sigma \in \mathcal{E}_{K}$, we denote by $x_{\sigma}$ the orthogonal projection of $x_{K}$ on $\mathcal{E}_{K}$ and by $d_{K \sigma}$ the Euclidean distance between $\mathbf{x}_{K}$ and $\mathbf{x}_{\sigma}$.

Time discretization: We divide the time interval $(0, T]$ into $N_{t}$ equal time steps of length $\delta t=T / N_{t}$, where $\delta t$ is the uniform time step defined by $\delta t=t_{n}-t_{n-1}$.

Let $u(\mathbf{x}, t)=(\rho(c) c)(\mathbf{x}, t)$ remembering that the function $\rho$ is strictly increasing. Since the function $u \rightarrow c$ is invertible, we can rewrite the concentration as a function of $u$, namely $c=r(u)$ and $\rho(c)=\rho(r(u))=R(u)$. For simplicity, for any function $v(\mathbf{x}, t)$, we use the notations $v^{n}(\mathbf{x})=v\left(\mathbf{x}, t_{n}\right)$ and $v_{K}^{n}=v\left(\mathbf{x}_{K}, t_{n}\right)$. 
ESAIM: PROCEEDINGS

We set $\theta(c)=\int_{0}^{c} \rho(s) d s$ so that $\Delta \theta(c)=\nabla \cdot(\rho(c) \nabla c)$ and define $\varphi(u)=\theta(c)=\theta(r(u))$. The first two equations of (1) then take the form

$$
\begin{cases}\Phi \frac{\partial u(\mathbf{x}, t)}{\partial t}+\nabla \cdot(\mathbf{q} u(\mathbf{x}, t))-D \Delta \varphi(u(\mathbf{x}, t))=0, & \text { in } \Omega \times(0, T], \\ \Phi \frac{\partial R(u)}{\partial t}+\nabla \cdot(\mathbf{q} R(u))=0, & \text { in } \Omega \times(0, T] .\end{cases}
$$

Next we present our numerical scheme which is based upon the standard finite volume method. In order to make this scheme more intuitive, we first formally integrate the two equations of System (5) on the domain $K \times\left(t_{n-1}, t_{n}\right)$ for each $K \in \mathcal{T}$ and $n=1, \ldots, N_{t}$ to obtain

$$
\begin{array}{r}
\int_{K} \Phi\left(u\left(\mathbf{x}, t_{n}\right)-u\left(\mathbf{x}, t_{n-1}\right)\right) d \mathbf{x}+\sum_{\sigma \in \mathcal{E}_{K}} \int_{t_{n-1}}^{t_{n}} \int_{\sigma}(\mathbf{q} u) \cdot \mathbf{n} d s d t-\sum_{\sigma \in \mathcal{E}_{K}} \int_{t_{n-1}}^{t_{n}} \int_{\sigma} D \nabla \varphi(u) \cdot \mathbf{n} d s d t=0 \\
\int_{K} \Phi\left(R \left(u\left(\mathbf{x}, t_{n}\right)-R\left(u\left(\mathbf{x}, t_{n-1}\right)\right) d \mathbf{x}+\sum_{\sigma \in \mathcal{E}_{K}} \int_{t_{n-1}}^{t_{n}} \int_{\sigma}(\mathbf{q} R(u)) \cdot \mathbf{n} d s d t=0 .\right.\right.
\end{array}
$$

Applying a semi-implicit scheme then yields

$$
\begin{array}{r}
\Phi \int_{K}\left(u^{n}-u^{n-1}\right) d \mathbf{x}+\delta t \sum_{\sigma \in \mathcal{E}_{K}} \int_{\sigma} \mathbf{q}^{n} u^{n} \cdot \mathbf{n} d s-\delta t \sum_{\sigma \in \mathcal{E}_{K}} \int_{\sigma} D \nabla \varphi\left(u^{n}\right) \cdot \mathbf{n} d s d t=0 \\
\int_{K} \Phi\left(R\left(u^{n-1}\right)-R\left(u^{n-2}\right)\right) d \mathbf{x}+\delta t \sum_{\sigma \in \mathcal{E}_{K}} \int_{\sigma}\left(\mathbf{q}^{n} R\left(u^{n-1}\right)\right) \cdot \mathbf{n} d s d t=0 .
\end{array}
$$

In view of Darcy's law, we define the discrete flux through an interior edge $\sigma=\sigma_{K L}$ between the elements $K$ and $L$ by

where

$$
F_{K L}\left(p^{m}, u^{n}\right)=\frac{k}{\mu}|\sigma|\left(\frac{p_{K}^{m}-p_{L}^{m}}{d_{K L}}+\mathbf{g} \cdot \mathbf{n}_{K L} R\left(\hat{u}_{K L}^{n}\right)\right),
$$

$$
\hat{u}_{K L}=\frac{u_{K}+u_{L}}{2} .
$$

We also introduce the upwind scheme for the convection term in equation (9)

$$
\tilde{u}_{K L}= \begin{cases}u_{K}, & \text { if } F_{K L}(p, u)>0 \\ u_{L}, & \text { otherwise }\end{cases}
$$

For the diffusion term, we define

$$
Q_{K L}\left(u^{n}\right)=-D \frac{\left|\sigma_{K L}\right|}{d_{K L}}\left(\varphi\left(u_{L}^{n}\right)-\varphi\left(u_{K}^{n}\right)\right)
$$

Therefore, a semi-implicit finite volume scheme corresponding to Problem (1) is given by the following equations

$$
\left.\begin{array}{c}
c_{K}^{0}=\frac{1}{|K|} \int_{K} c_{0}(\mathbf{x}) d \mathbf{x}, \quad u_{K}^{0}=\rho\left(c_{K}^{0}\right) c_{K}^{0}, \\
\frac{\Phi|K|}{\delta t}\left(R\left(u_{K}^{n-1}\right)-R\left(u_{K}^{n-2}\right)\right)+\sum_{L \in \mathcal{N}_{K}} F_{K L}\left(p^{n}, u^{n-1}\right) R\left(\hat{u}_{K L}^{n-1}\right)=0, \\
\frac{\Phi|K|}{\delta t}\left(u_{K}^{n}-u_{K}^{n-1}\right)+\sum_{L \in \mathcal{N}_{K}} F_{K L}\left(p^{n}, u^{n-1}\right) \tilde{u}_{K L}^{n}+\sum_{L \in \mathcal{N}_{K}} Q_{K L}\left(u^{n}\right)=0,
\end{array}\right\}
$$


where the convection term $F_{K L}$ is defined by (10) and the diffusion term $Q_{K L}$ is defined by (13). Note that when $n=1$, the first term of the first equation in System (15) is omitted.

\section{HENRY'S PROBLEM}

Henry's problem describes the advance of a salt water front in a confined aquifer which is initial charged with fresh water. Henry developed a solution method to compute the steady-state distribution of the solute. To this end, he applied the Boussinesq approximation, which involves a stream function. Henry derived analytical expressions for the stream function and the concentration in the form of a Fourier series, the resulting algebraic equations for the determination of the Fourier coefficients must be obtained numerically. The 'mystery' of Henry's problem is that no numerical model so far has been able to closely reproduce his semi-analytical solution. Nevertheless, because of the absence of other non numerical solution for this kind of nonlinear problems, Henry's solution has become one of the standard tests for variable density groundwater models.

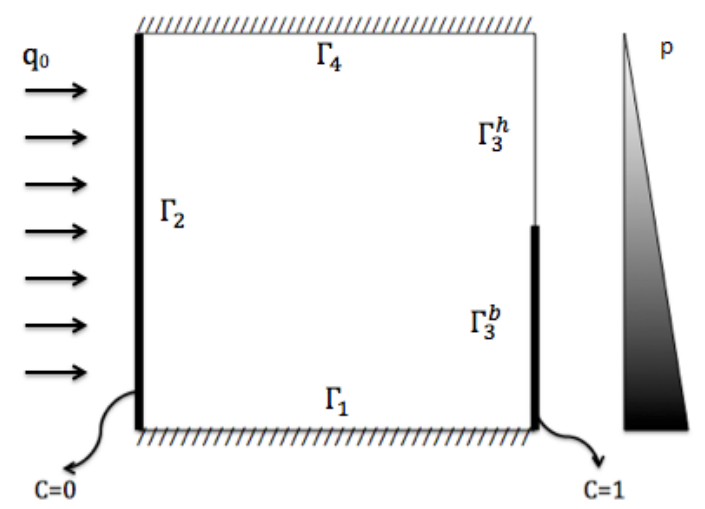

Figure 1. The various parts of the boundary for Henry's problem.

Mathematically, Henry's problem is defined as System (1) in the rectangle $\Omega$ together with the boundary conditions

$$
\begin{cases}c=0, & \text { over } \Gamma_{2} \times(0, T], \\ c=1, & \text { over } \Gamma_{3}^{b} \times(0, T], \\ \frac{\partial c}{\partial n}=0, & \text { on }\left(\Gamma_{1} \cup \Gamma_{4} \cup \Gamma_{3}^{h}\right) \times(0, T], \\ p=\rho_{0}|\mathbf{g}|(\alpha(1-y)-y), & \text { on } \Gamma_{3} \times(0, T], \\ \mathbf{q} \cdot \mathbf{n}=0, & \text { on }\left(\Gamma_{1} \cup \Gamma_{4}\right) \times(0, T], \\ \mathbf{q} \cdot \mathbf{n}=q_{0}, & \text { on } \Gamma_{2} \times(0, T],\end{cases}
$$

where $\Gamma_{3}=\Gamma_{3}^{h} \cup \Gamma_{3}^{b}$ and $y$ is $y$-coordinate. Figure 1 shows the configuration of the boundary conditions. The initial conditions are such that $c(\mathbf{x}, 0)=0$ and that the pressure $p(\mathbf{x}, 0)$ satisfies $\nabla \cdot(\mathbf{q} \rho(c))=0$ for all $\mathbf{x} \in \Omega$.

We perform the numerical tests on the space domain $\Omega=(0,1) \times(0,1)$, with $T=0.05$ (day). We use $20 \times 20$ square control volumes in $\Omega$. The parameters are given in Table 1 . In our study, the unit length, the unit time and the unit mass are chosen as the meter, the second and the kilogram respectively. 


\begin{tabular}{|l|c|r|}
\hline Symbol & Value & Unit \\
\hline$k$ & $1.02 \times 10^{-9}$ & $\mathrm{~m}^{2}$ \\
\hline$D$ & $6.6 \times 10^{-6}$ & $\mathrm{~m}^{2} . \mathrm{s}^{-1}$ \\
\hline $\mathbf{g}$ & 9.81 & $\mathrm{~m} . \mathrm{s}^{-2}$ \\
\hline$\Phi$ & 0.3 & - \\
\hline$\mu$ & $10^{-3}$ & Pa.s \\
\hline$\rho_{0}$ & $10^{3}$ & $\mathrm{~kg} . \mathrm{m}^{-3}$ \\
\hline$\alpha$ & 0.025 & - \\
\hline$q_{0}$ & $-6.6 \times 10^{-5}$ & $\mathrm{~m} . \mathrm{d}^{-1}$ \\
\hline
\end{tabular}

TABlE 1. Parameter values for Henry's problem.
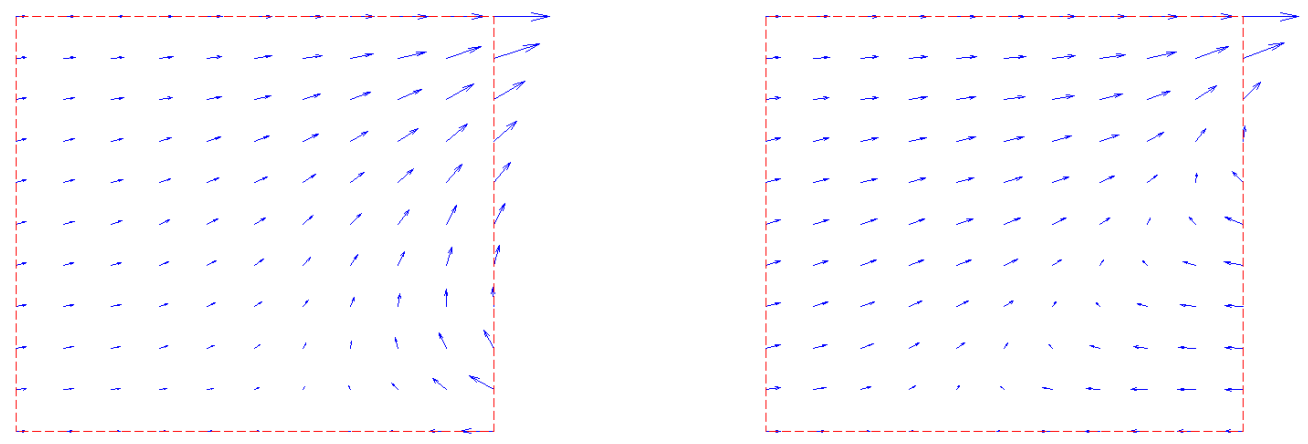

Figure 2. The velocity field at the initial and the end times for Henry's problem.

The simulations describe the intrusion of salt water in a confined aquifer. The salt water enters from the right-hand-side, while the fresh water, of density $\rho_{0}$, flows in from the left-hand-side at a constant rate. Therefore, the concentration in the area near the coastal side increases in time. At first the interface between the fresh and the salt water coming from the right-bottom corner has a large slope, which slowly decreases in time while the salt concentration enters deeper in the domain. Figure 2 shows the velocity fields at the initial and at the end times.

The simulations in the figures 3 - 4 show the comparison between our numerical results and those obtained with the FEflow software. FEflow, which is developed by the German group DHI-WASY GmbH, is a professional software package for modeling fluid flow, transport of dissolved constituents and heat transport processes in the subsurface. It contains pre- and post processing functionalities together with an efficient simulation engine. A user-friendly graphical interface provides easy access to a number of modeling options.

\section{The Rotating Interface Problem}

We now consider the case of a rotating interface between fresh and salt water. The salt water, which is heavier than the fresh water, has a tendency to flow to the bottom, while the fresh water flows to the top. At the initial time, the space domain is divided into two rectangles as shown in Figure 5. The left rectangle is filled with salt water while the right one is filled with fresh water. The interface between these two liquids rotates 

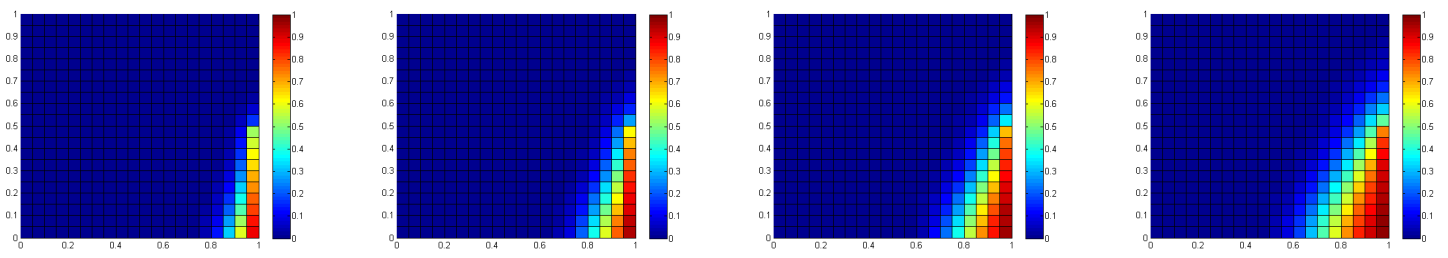

FVM $t=0.001$ days $t=0.002$ days

$\mathrm{t}=0.003$ days $\mathrm{t}=0.005$ days
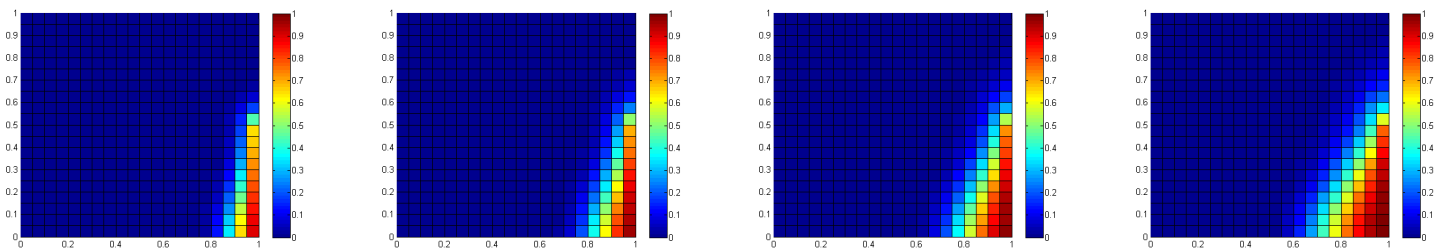

FEflow $\mathrm{t}=0.001$ days $\mathrm{t}=0.002$ days $\mathrm{t}=0.003$ days $\mathrm{t}=0.005$ days

FiguRE 3. Fluid concentration from $t=0.001$ to $t=0.005$ days for Henry's problem.
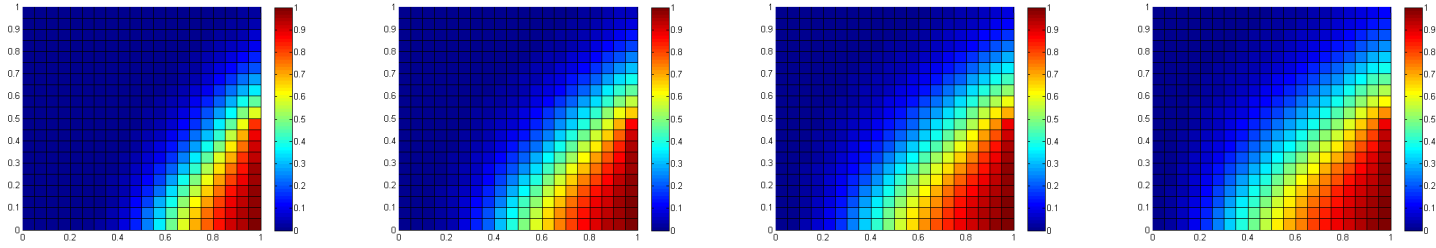

FVM $t=0.01$ days $t=0.02$ days $\mathrm{t}=0.03$ days $\quad \mathrm{t}=0.05$ days
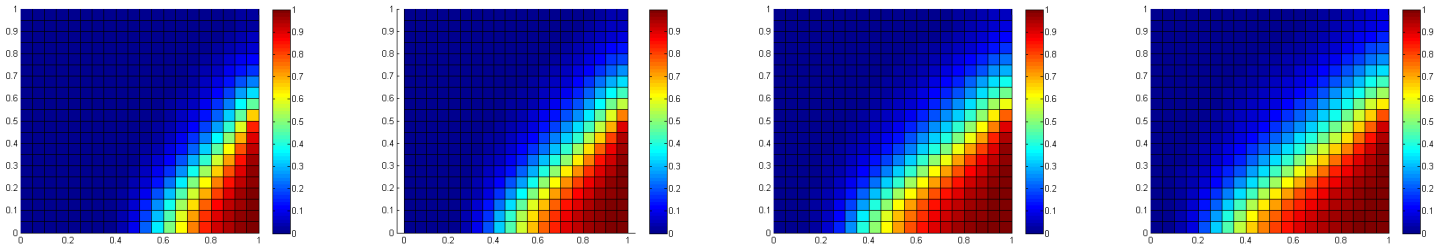

FEflow $\mathrm{t}=0.01$ days $\mathrm{t}=0.02$ days $\mathrm{t}=0.03$ days $\mathrm{t}=0.05$ days

FiguRE 4. Fluid concentration from $t=0.01$ to $t=0.05$ days for Henry's problem.

counterclockwise until it becomes horizontal. The simulations described below permit to follow the rotation of the interface.

We suppose that $c$ and $\mathbf{q}$ satisfy the boundary conditions:

$$
\begin{cases}\frac{\partial c}{\partial n}=0, & \text { on } \partial \Omega \times(0, T], \\ \mathbf{q} \cdot \mathbf{n}=0, & \text { on } \partial \Omega \times(0, T] .\end{cases}
$$

A technical problem is that if $p$ is a solution, also $p+c$ is a solution, with $c$ an arbitrary constant. This leads us to transform the elliptic equation for $p$ into a parabolic equation by adding the extra term $\varepsilon \frac{\partial p(\mathbf{x}, t)}{\partial t}$ to the pressure equation, which makes the model slightly compressible. In literature, this is referred to as a 
pseudo-compressibility approximation (cf. [3]). The second equation in Problem (1) becomes

$$
\epsilon \frac{\partial p}{\partial t}+\Phi \frac{\partial R(u)}{\partial t}+\nabla \cdot(\mathbf{q} R(u))=0
$$

Therefore, the semi-implicit finite volume scheme corresponding to this new problem is given by:

$$
\begin{gathered}
c_{K}^{0}=\frac{1}{|K|} \int_{K} c_{0}(\mathbf{x}) d \mathbf{x}, \quad u_{K}^{0}=\rho_{K}^{0} c_{K}^{0}, \\
\left.p_{K}^{0}=\frac{1}{|K|} \int_{K} p_{0}(\mathbf{x}) d \mathbf{x}, \quad \text { for all } K \in \mathcal{T}, \quad\right\} \\
\frac{\epsilon|K|}{\delta t}\left(p_{K}^{n}-p_{K}^{n-1}\right)+\frac{\Phi|K|}{\delta t}\left(R\left(u_{K}^{n-1}\right)-R\left(u_{K}^{n-2}\right)\right)+\sum_{L \in \mathcal{N}_{K}} F_{K L}\left(p^{n}, u^{n-1}\right) R\left(\hat{u}^{n-1}\right)=0, \\
\frac{\Phi|K|}{\delta t}\left(u_{K}^{n}-u_{K}^{n-1}\right)+\sum_{L \in \mathcal{N}_{K}} F_{K L}\left(p^{n}, u^{n}\right) \tilde{u}_{K L}^{n}+\sum_{L \in \mathcal{N}_{K}} Q_{K L}\left(u^{n}\right)=0 .
\end{gathered}
$$

\subsection{Numerical results}

We take as space domain the domain $\Omega=(0,100) \times(0,100)$ and consider the time interval $(0, \mathrm{~T})$ with $T=500$ (days). We use $21 \times 21$ square control volumes. The values of the parameters are given in Table 2 and the time evolution of the densities is plotted in the figures 6 and 7 .

\begin{tabular}{|l|c|r|}
\hline Symbol & Value & Unit \\
\hline$k$ & $3.10 \times 10^{-12}$ & $\mathrm{~m}^{2}$ \\
\hline$D$ & $3.3 \times 10^{-6}$ & $\mathrm{~m}^{2} . \mathrm{s}^{-1}$ \\
\hline $\mathbf{g}$ & 9.81 & $\mathrm{m.s}^{-2}$ \\
\hline$\Phi$ & 0.5 & - \\
\hline$\mu$ & $10^{-3}$ & Pa.s \\
\hline$\rho_{0}$ & $10^{3}$ & $\mathrm{kg.m}$ \\
\hline$\alpha$ & 0.3 & - \\
\hline$\varepsilon$ & $10^{-5}$ & - \\
\hline
\end{tabular}

TABLE 2. Parameter values for the rotating interface problem.
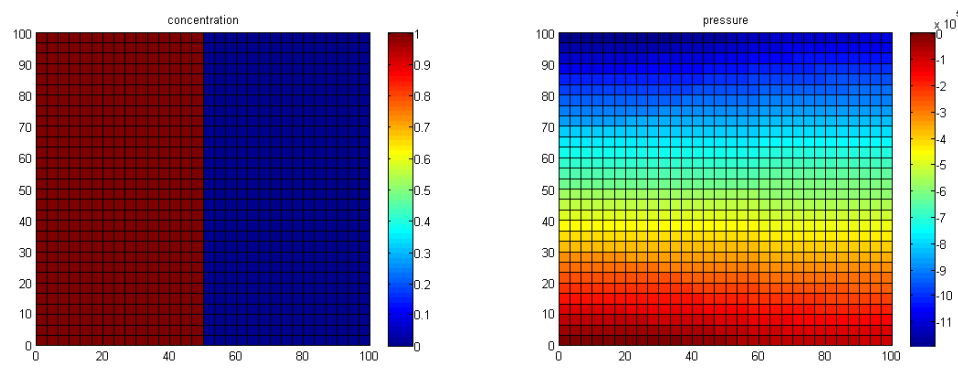

FIGURE 5. Initial conditions for concentration and pressure.

The initial concentration is given by $c(\mathbf{x}, 0)=0$ if $\mathbf{x}$ belongs the right half domain and $c(\mathbf{x}, 0)=1$ otherwise. The pressure $p$ is such that $p(\mathbf{x}, 0)=0$ at $\mathbf{x}_{0}=(0,0)$ and satisfies $\nabla \cdot(\mathbf{q} \rho(c))=0$ for all $\mathbf{x} \in \Omega \backslash \mathbf{x}_{0}$. Figure 5 
shows the functions $c(\mathbf{x}, 0)$ and $p(\mathbf{x}, 0)$.

The salt water diffuses from left to right. Under the influence of gravity, the heavier fluid (the salt water) goes down while the light fluid (fresh water or slightly salted water) goes up. Therefore, the interface slowly moves from a vertical to a horizontal line. After a long time (about 500 days), the fluid is in balance and the motion stops.

Our method and the FEflow software first give very similar results while they are slightly different for very large times $t$ (around 200 - 500 days). Our numerical results are plotted above and the pictures plotted below are obtained with FEflow.
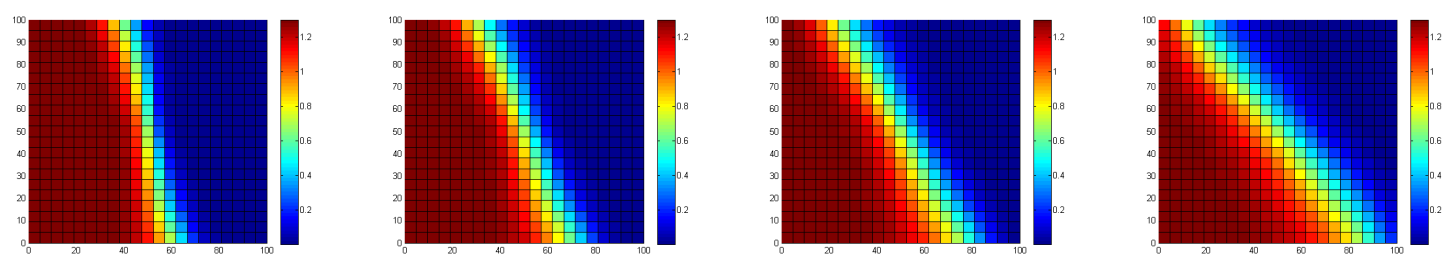

FVM $\mathrm{t}=10$ days $\mathrm{t}=20$ days $\mathrm{t}=30$ days $\mathrm{t}=50$ days
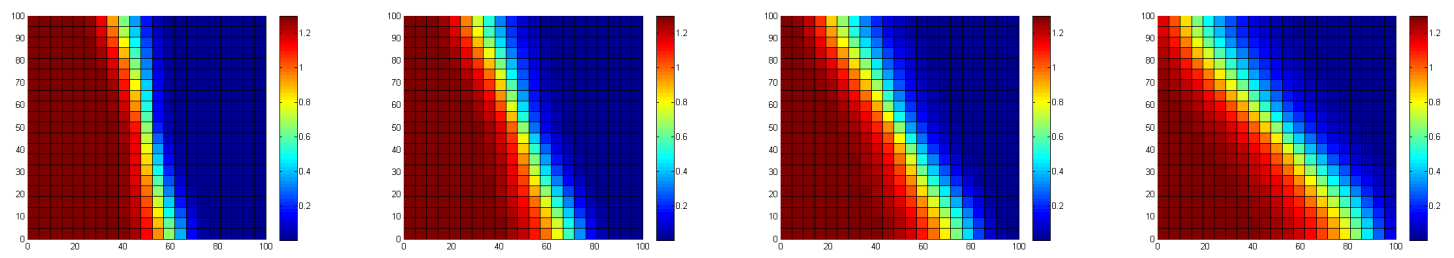

FEflow $\mathrm{t}=10$ days $\mathrm{t}=20$ days $\mathrm{t}=30$ days $\mathrm{t}=50$ days

Figure 6. Comparison of the results from $t=10$ to $t=50$ days.
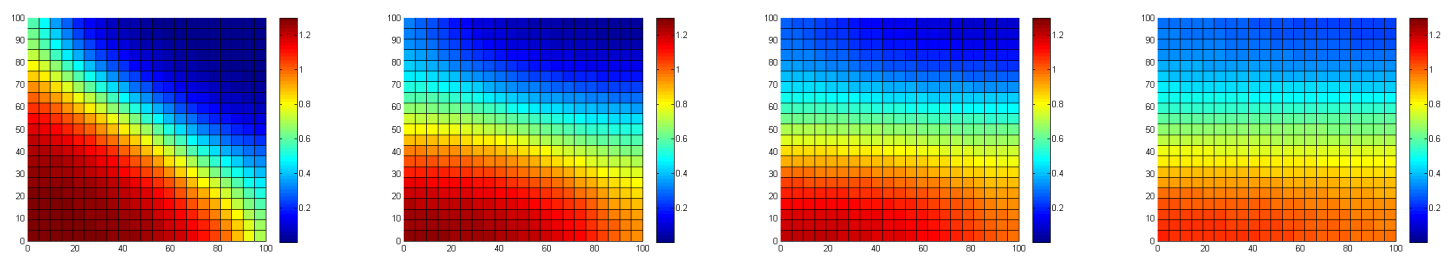

FVM $\mathrm{t}=100$ days $\mathrm{t}=200$ days $\mathrm{t}=300$ days $\mathrm{t}=500$ days
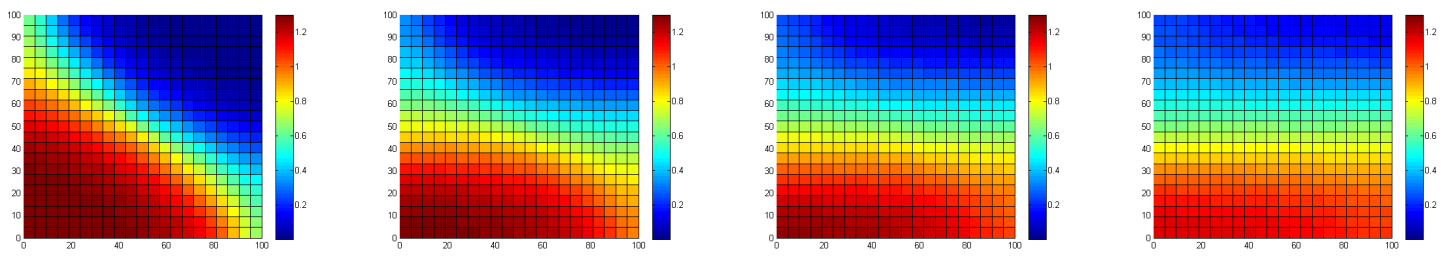

FEflow $t=100$ days $t=200$ days $t=300$ days $t=500$ days

Figure 7. Comparison of the results from $t=100$ to $t=500$ days. 
Table 3 shows the error in the concentration between two methods. We define the error as

$$
e=\frac{\left\|C_{F V M}-C_{F E \text { flow }}\right\|_{L^{2}(\Omega)}}{\left\|C_{F E \text { flow }}\right\|_{L^{2}(\Omega)}}
$$

where $C_{F V M}$ is the concentration obtained by our method and $C_{F E \text { flow }}$ is the result from the FEflow program.

\begin{tabular}{|l|c|}
\hline time & mesh 31x31 \\
\hline$t=0$ & 0 \\
\hline$t=10$ & 0.0155 \\
\hline$t=20$ & 0.0192 \\
\hline$t=30$ & 0.0219 \\
\hline$t=50$ & 0.0271 \\
\hline$t=100$ & 0.0379 \\
\hline$t=200$ & 0.0495 \\
\hline$t=300$ & 0.0660 \\
\hline$t=500$ & 0.1061 \\
\hline
\end{tabular}

TABLE 3. Error between the solutions obtained by the finite volume method and by FEflow.

Next we discuss the conservation of mass. For each $t$, we integrate the transport equation on $\Omega \times[0, t]$ :

$$
\begin{array}{r}
\int_{\Omega} \Phi\left(\rho(c(\mathbf{x}, t)) c(\mathbf{x}, t)-\rho\left(c_{0}(\mathbf{x})\right) c_{0}(\mathbf{x})\right) d \mathbf{x}+\int_{0}^{t} \int_{\Omega} \nabla \cdot\left(\mathbf{q} \rho\left(c\left(\mathbf{x}, t^{\prime}\right)\right) c\left(x, t^{\prime}\right)\right) d x d t^{\prime} \\
-D \int_{0}^{t} \int_{\Omega} \triangle \theta\left(c\left(\mathbf{x}, t^{\prime}\right)\right) d \mathbf{x} d t^{\prime}=0
\end{array}
$$

which yields

$$
\begin{aligned}
\int_{\Omega} \Phi \rho(c(\mathbf{x}, t)) c(\mathbf{x}, t) d \mathbf{x}= & \int_{\Omega} \Phi \rho\left(c_{0}(\mathbf{x})\right) c_{0}(\mathbf{x}) d \mathbf{x}-\int_{0}^{t} \int_{\partial \Omega} \rho\left(c\left(\mathbf{x}, t^{\prime}\right)\right) c\left(\mathbf{x}, t^{\prime}\right) \mathbf{q} \cdot \mathbf{n} d \mathbf{x} d t^{\prime} \\
& +D \int_{0}^{t} \int_{\partial \Omega} \nabla \theta\left(c\left(\mathbf{x}, t^{\prime}\right)\right) \cdot \mathbf{n} d \mathbf{x} d t^{\prime}
\end{aligned}
$$

In view of the boundary conditions (17) we obtain

$$
\int_{\Omega} \Phi \rho(c(\mathbf{x}, t)) c(\mathbf{x}, t) d \mathbf{x}=\int_{\Omega} \Phi \rho\left(c_{0}(\mathbf{x})\right) c_{0}(\mathbf{x}) d \mathbf{x} .
$$

Similarly summing the second equation in (20) yields

$$
\sum_{K \in \mathcal{M}}|K| \rho_{K}^{n} c_{K}^{n}=\sum_{K \in \mathcal{M}}|K| \rho_{K}^{0} c_{K}^{0}
$$

which expresses the fact that the finite volume method exactly conserves the mass. 


\begin{tabular}{|l|c|c|}
\hline time & FVM & FEflow \\
\hline$t=0$ & $6.5 \times 10^{6}$ & $6.5 \times 10^{6}$ \\
\hline$t=10$ & $6.5 \times 10^{6}$ & $6.5 \times 10^{6}$ \\
\hline$t=20$ & $6.5 \times 10^{6}$ & $6.5 \times 10^{6}$ \\
\hline$t=30$ & $6.5 \times 10^{6}$ & $6.5 \times 10^{6}$ \\
\hline$t=50$ & $6.5 \times 10^{6}$ & $6.5 \times 10^{6}$ \\
\hline$t=100$ & $6.5 \times 10^{6}$ & $6.5 \times 10^{6}$ \\
\hline$t=200$ & $6.5 \times 10^{6}$ & $6.5 \times 10^{6}$ \\
\hline$t=300$ & $6.5 \times 10^{6}$ & $6.5 \times 10^{6}$ \\
\hline$t=500$ & $6.5 \times 10^{6}$ & $6.5 \times 10^{6}$ \\
\hline
\end{tabular}

TABLE 4. Total mass with the finite volume method and with FEflow.

\section{Conclusion}

Both our finite volume code and the FEflow package provide efficient computations for the density driven flow problem which we have been studying. Possibly our code is slightly more diffusive. Future work will involve a recently developed finite volume scheme such as SUSHI, which will permit to also consider cases that the diffusion constant $D$ and the permeability $k$ are replaced by full diffusion tensors [1].

Acknowledgments. The authors are very grateful for the support of François Bertone (EGIS) and Marc Bonnet (SHYGMA). They also acknowledge fruitful discussions with Roland Masson (University of Nice). They thank CNRS for the PEPS ECODEVA grant which has permitted them to carry out this project, and the DHI-WASY Company who has granted them a full access to the FEflow Software.

\section{REFERENCES}

[1] Ophélie Angelini, Konstantin Brenner, Danielle Hilhorst. A finite volume method on general meshes for a degenerate parabolic convection-reaction-diffusion equation, To appear in Numerische Mathematik.

[2] Peter Bastian, Klaus Johannsen, Stefan Lang, Christian Wieners, Volmer Reichenberger, Gabriel Wittum. High-accuracy simulation of density driven flow in porous media, prepublication.

[3] Alexandre Joel Chorin. A numerical method for solving incompressible viscous flow problems, J. Comp. Phys. 135 (1997), 118-125.

[4] M. Dentz, D.M. Tartakovsky, E. Abarca, A. Guadagnini, X. Sanchez-Vila, J. Carrera. Variable-density flow in porous media, J. Fluid Mech. 561 (2006), 209-235.

[5] Hans-Jörg G. Diersch, Olaf. Kolditz. Variable-density flow and transport in porous media: approches and challenges, Advances in Water Resources 25 (2002), 899-944.

[6] Robert Eymard, Thierry Gallouët, Danielle Hilhorst, Sabrina Naït Slimane. Finite volumes and non linear diffusion equations, RAIRO Modél. Math. Anal. Numér. 32 (1998), 747-761.

[7] Robert Eymard, Thierry Gallouët, Mustapha Ghilani, Raphaèle Herbin. Error estimates for the approximate solutions of a nonlinear hyperbolic equation given by some finite volumes schemes, IMA J. Numer. Anal. 18 (1998), 563-594.

[8] Robert Eymard, Thierry Gallouët, Raphaèle Herbin, Michaël Gutnic, Danielle Hilhorst. Approximation by the finite volume method of an elliptic-parabolic equation arising in environmental studies, Mathematical Models and Methods in Applied Sciences, Vol. 11, No. 9 (2001) 1505-1528.

[9] Robert Eymard, Thierry Gallouët, Raphaèle Herbin, Anthony Michel. Convergence of a finite volume scheme for nonlinear degenerate parabolic equation, Numer. Math. 92 (2002), 41-82.

[10] Peter Knabner, Christoph Tapp, Kathrin Thiele. Adaptive Finite Volume Discretization of Density Driven Flows in Porous Media, Acta Math. Univ. Comenian. (N.S.) 67 (1998), 115-136.

[11] Olaf Kolditz, Rainer Ratke, Hans-Jörg G. Diersch, Werner Zielke. Coupled groundwater flow and transport: 1. Verification of variable density flow and transport models, Advances in Water Resources 21 (1998), 27-46. 